\title{
Express delivery
}

A recent report in Molecular Cell shows that retroviral vectors can be used to deliver DNA and protein in an efficient, dose-controlled, transient and targeted manner. It had been proposed that pseudotransduction - transduction without proviral DNA integration into the host genome - could be used to express exogenous DNA. To explore this possibility, Christopher Baum and colleagues modified mouse leukaemia virus in two ways: they blocked proviral DNA integration by preventing reverse transcription of the viral RNA and inserted a nucleus-targeted site-specific recombinase Cre gene into the viral genome.

Using mouse and human fibroblasts that are engineered to express EGFP only on CRE-mediated recombination, the authors confirmed that these viral particles can

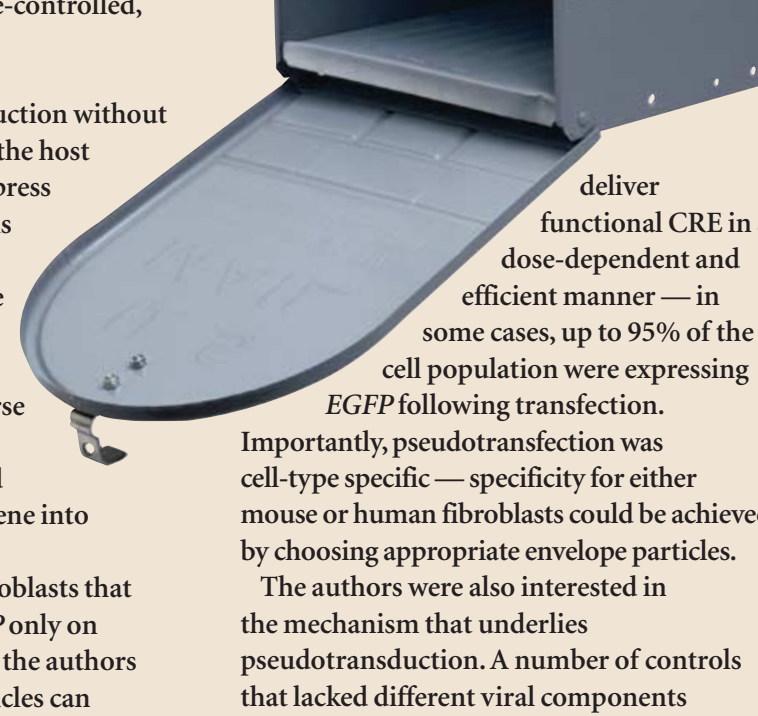

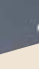
(1) peudotranscription, the authors sugges that this process could be extended to other viruses and propose a new term for it particle-mediated mRNA transfer (PMT).

The ability to express Cre in a transient and targeted manner is useful in itself, but the authors have a more ambitious vision. They suggest that PMT could be particularly useful in cases where low and transient expression might lead to striking biological results, such as for the expression of receptors that are involved in the homing of circulating cells or transcription factors.

\section{Magdalena Skipper}

\section{(9) References and links}

ORIGINAL RESEARCH PAPER Galla, M. \& Will, E. et al. Retroviral pseudotransduction for targeted cell manipulation. Mol. Cell 16, 309-315 (2004)

\section{CANCER GENETICS}

\section{Second-generation models}

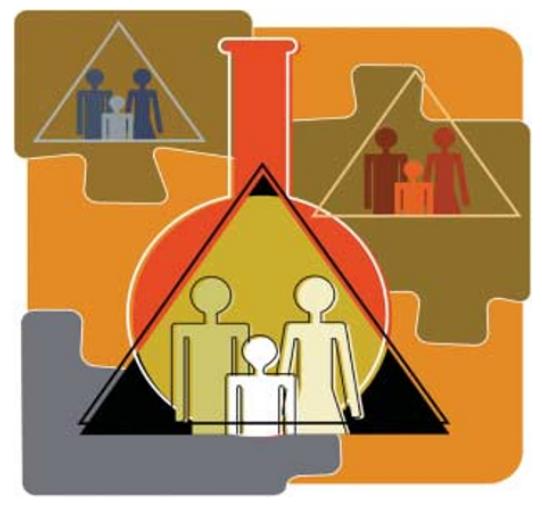

The production of mouse models to study particular human cancers has become something of a cottage industry. The difficulty in exploiting them arises from the underlying genetic heterogeneity of most cancers, which means that the molecular pathways that are disrupted in a particular mouse might be irrelevant to the human disease, despite an overall phenotypic similarity. A study in Nature Genetics addresses this issue by outlining a new method to identify the best-fit mouse model for hepatocellular carcinoma (HCC).
HCC is the fifth most common cancer in the world, causing at least 500,000 deaths annually. Although only three agents - hepatitis B virus, hepatitis $\mathrm{C}$ virus and aflatoxin $\mathrm{B} 1$ - are responsible for approximately $80 \%$ of all HCCs in humans, the genetic and epigenetic changes that follow exposure are varied and incompletely understood. Snorri Thorgeirsson and colleagues describe a comparative functional-genomic approach that compares the similarities in gene expression between 68 HCCs from 7 mouse models and 91 human HCCs from pre-defined sub-classes. The mouse models include two chemically induced, four transgenic and one knockout line.

The authors first applied hierarchical clustering analysis to previously generated microarray data from the mouse HCCs and identified three distinct groups. Using a subset of the genes that they had previously shown to be associated with survival in human HCC, they compared their expression between the three subgroups of mouse HCC and two subclasses of human HCC. In this clustering analysis, three of the transgenic mouse lines with targeted overexpression in the liver (Myc, E2f1 and Myc/E2f1) had the highest relative similarity to those in the better survival group of human HCC, whereas the $M y c / T g f a$ transgenic and one of the chemically induced models were most similar to the poor survival group of human HCC. Thorgeirsson and co-workers then found that the individual genes that differentiated the two human HCC subclasses showed a highly similar differential expression in the mouse models that compare best with them.

Do the similarities at the transcriptional level reflect phenotypic similarities between the mouse and human HCCs? By several criteria, the answer is yes. For example, the Myc/Tgfa line has a poor prognosis phenotype, with a higher rate of HCC development and higher mortality. Ultimately, this cross-species approach that is based on overall patterns of gene expression might help to integrate mouse models of cancer with mainstream cancer research more rapidly, and should enable the identification of new mechanisms and treatments.

Alan Packer, Senior Editor, Nature Genetics

6) References and links

ORIGINAL RESEARCH PAPER Lee, J.-S. et al. Application

of comparative functional genomics to identify best-fit mouse models to study human cancer. Nature Genet. 21 November 2004 (doi:10.1038/ng1481) WEB SITE

Snorri Thorgeirsson's laboratory: http://neoplasia.nci.nih.gov/ 